\section{Nanoparticle Measurement Through Visualisation}

\author{
Bob Carr and Andrew Malloy \\ NanoSight, Ltd. Wiltshire, U.K. \\ andrewmalloy@nanosight.co.uk
}

\section{Introduction}

In this article, we will describe how nanoscale particles may be individually visualised (but not imaged) in liquids and from which higher resolution particle size distribution profiles can be obtained compared to other light scattering techniques. The method is called Nanoparticle Tracking Analysis (NTA)

Sample preparation is minimal, requiring only dilution with a suitable solvent to an acceptable concentration range (between 10 and 10 particles per $\mathrm{ml}$ depending on sample type). Accurate and reproducible analyses can be obtained from video images of only a few seconds duration and the results allow particle number concentration to be recovered. Given the close to real-time nature of the technique, particle-particle interactions are accessible as is information about sample aggregation and dissemination. All particle types can be measured and in any solvent type providing that the particles scatter sufficient light to be visible (i.e. are not indexed matched).

The technique is robust and low cost representing an attractive alternative or complement to some of the higher cost and more complex methods of nanoparticle analysis such as photon correlation spectroscopy or electron microscopy.

This technology uniquely allows the user a simple and direct qualitative view of the sample under analysis (perhaps to validate data obtained from other techniques such as PCS) and from which an independent quantitative estimation of sample size, size distribution and concentration can be immediately obtained.

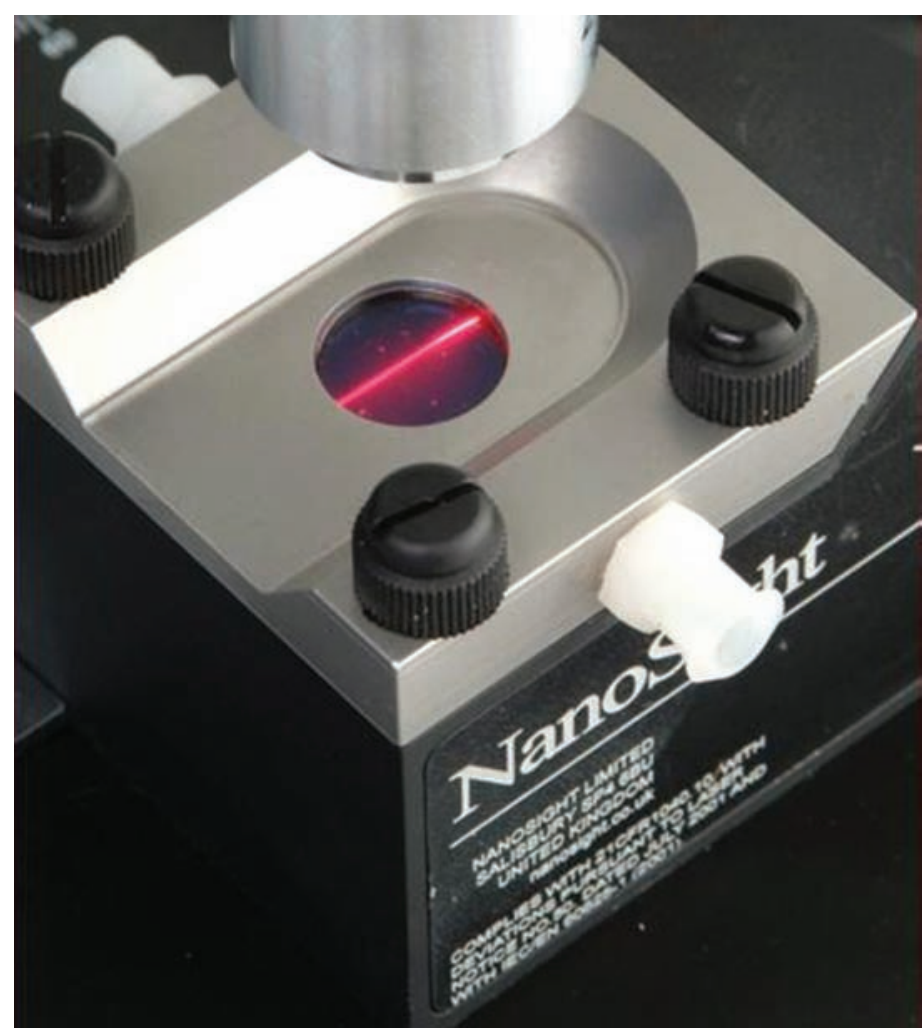

Figure 1, Flow Cell

\section{How does NTA work?}

NTA is a unique method to visualise and analyse particles in liquids relating the rate of Brownian motion to particle size. The rate of this movement is related only to the viscosity of the liquid, the temperature and size of the particle and is not influenced by particle density. NTA enables individual particles to be studied rather than the averaging techniques employed by PCS, also known as Dynamic Light Scattering (DLS).

The size range applicable to NTA is from 10 to $1,000 \mathrm{~nm}$, depending on the particle type. Analysis of $10 \mathrm{~nm}$ particles is only possible for particles with a high refractive index such as gold and silver while the upper size limit is restricted by the limited Brownian motion. At 1 micron, particles move very slowly and hence the accuracy of the technique starts to diminish. The viscosity of the solvent also plays a role in determining the upper size limit as it too influences the movement of the particles.

Populations of particles are studied rather than a search for one or two contaminant particles in a big volume. In the ideal measurement, a concentration of $10^{6}-10^{9}$ particles per $\mathrm{ml}$ is used. This equates to less than $1 \mathrm{wt} \%$ which means that for some applications, the sample needs to be diluted. Care should be taken when diluting a sample as this may sometimes lead to particle aggregation.

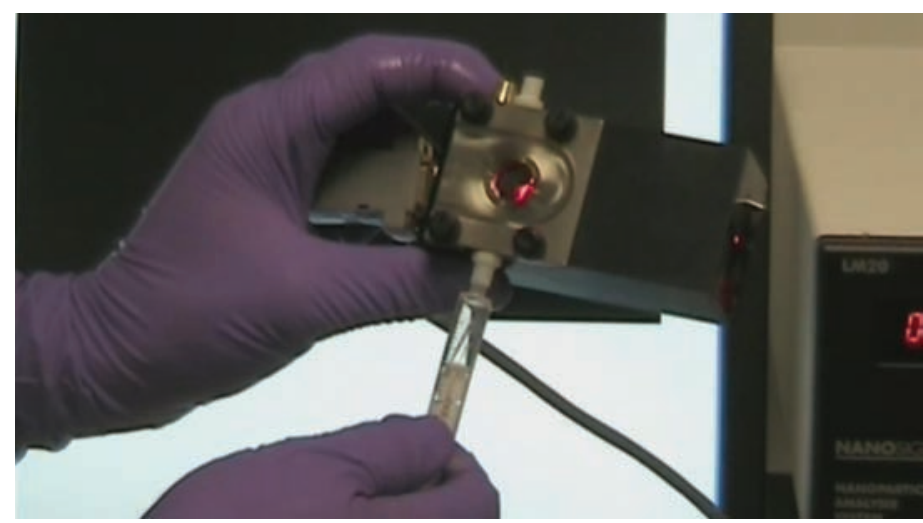

Figure 2, Injecting sample into the cell.

\section{What hardware is required?}

NTA employs a specially designed flow cell, figure 1 , that is mounted on a conventional optical microscope equipped with a CCD camera capable of operating at 30 frames per second.

A suitably prepared sample of nanoparticles in solution is injected into the cell, figure 2 . The beam from a laser diode is passed through the liquid sample cell.

Particles in the beam produce light scattering that is viewed with a suitable optical microscope and CCD camera against a proprietary metallised surface, figure 3 .

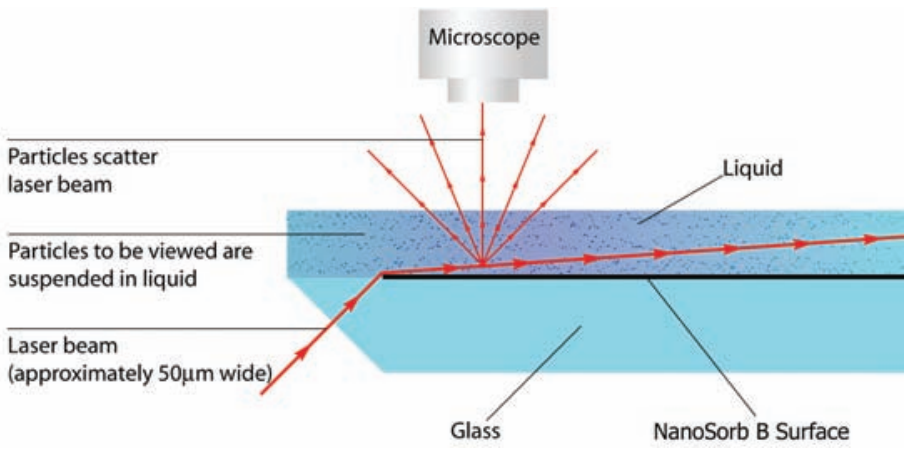

Figure 3, Schematic of the system 
EMS offers the breakthrough solutions of QuantomiK's propriatary WETSEM ${ }^{\circledR}$ Technology now available at heilf price for a limiled time
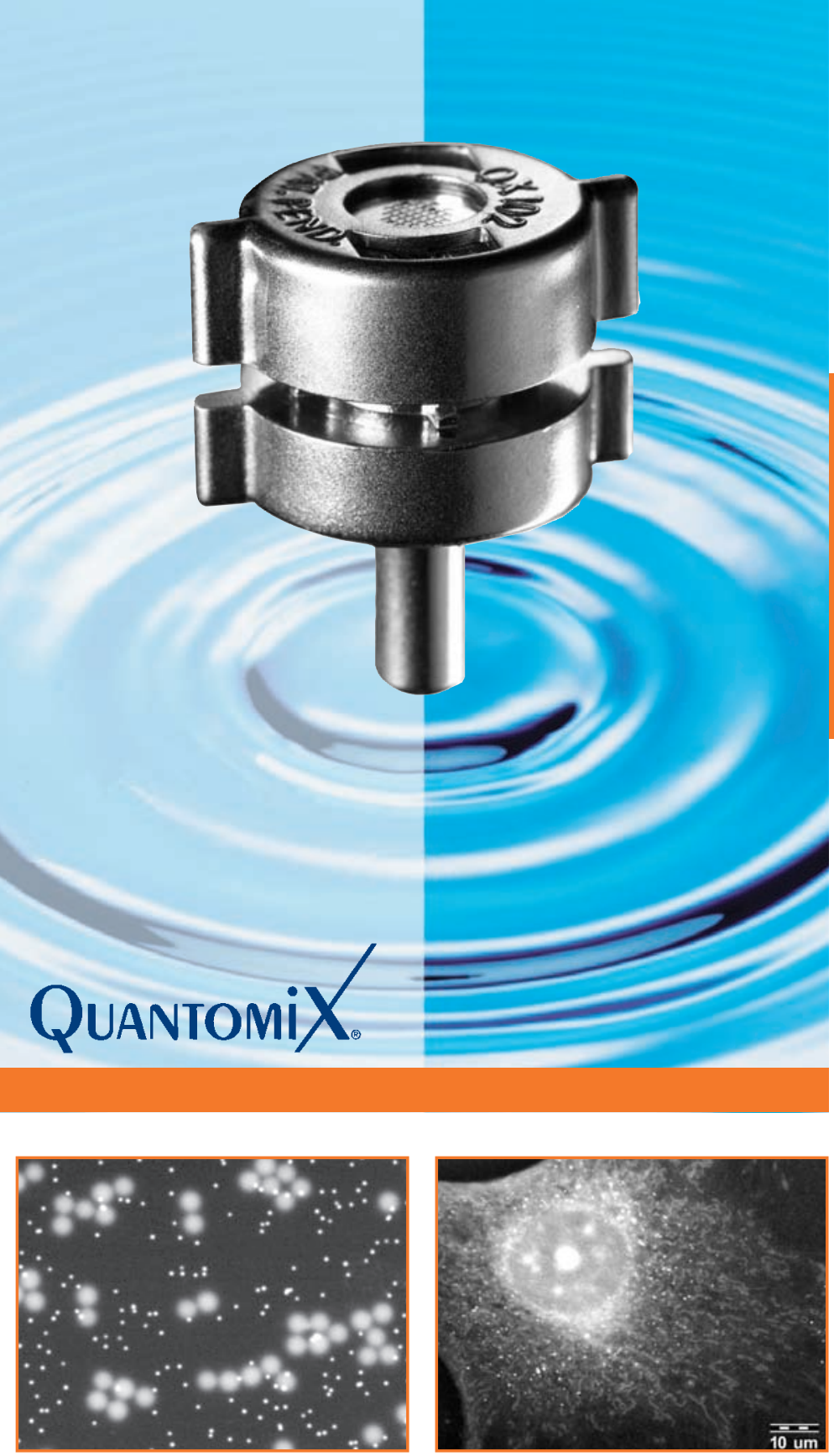

Silica $(500 \mathrm{~nm})$ and gold $(40 \mathrm{~nm})$ particles

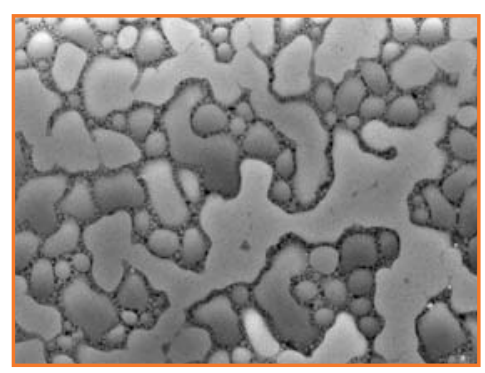

Volatile deodorant showing phase separation

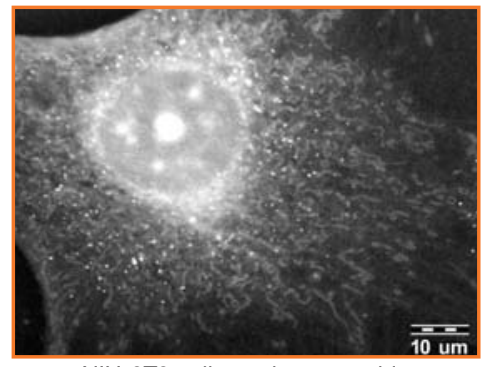

NIH-3T3 cell, osmium tetroxide

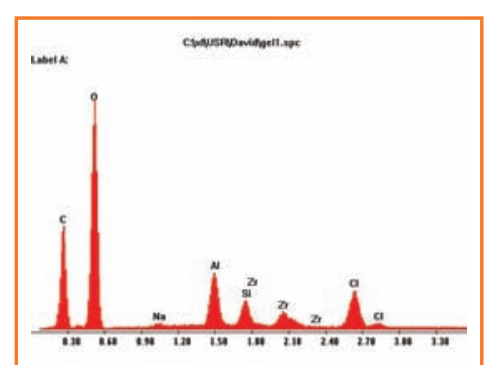

EDS graph showing deodorant material composition
The biggest breakthrough is here in a nano-package.

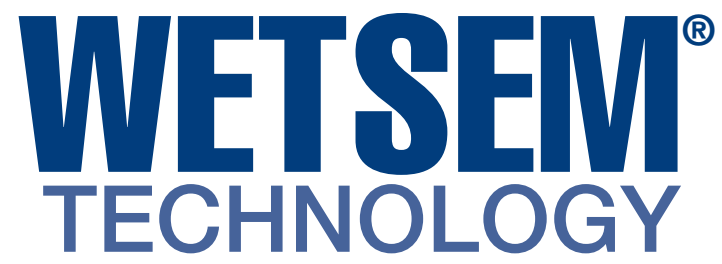

Attain EM-level imaging of wet samples

Reduce artifacts \& time-consuming sample preparation

Safe and simple to use

Achieve reliable, reproducible \&

quantifiable results

\section{Applications}

- Industrial research: food, oils, dyes, emulsions, pharmaceuticals, suspensions, personal care goods, cosmetics, inks.

- Quality control and quality assurance.

- Life sciences and medicine: cultured and primary cells, histology, nerve cells and myelin imaging, microbiology, plants.

- Tissue implants and prostheses.

- Environmental and toxicological applications.

- Clinical diagnosis: histopathology, cytology, oncology.

\section{Capabilities}

- Analyze size distribution, aggregation and homogeneity of particle suspensions.

- Perform EDS of fully hydrated samples.

- SEM imaging of oils, greases, volatile substances, emulsions and creams.

- Enables tissue morphology and analysis.

- Characterize cellular and subcellular organelles, cell contacts and receptors, cytoskeleton and other detail.

- Characterize and quantify lipids in fully-wet cells and tissues.

Please contact us for more details.

\section{Electron Microscopy Sciences}

In PA: (215) 412-8400 • Toll-Free (800) 523-5874

Fax (215) $412-8450$ or 8452

email: sgkcck@aol.com or stacie@ems-secure.com

www.emsdiasum.com 

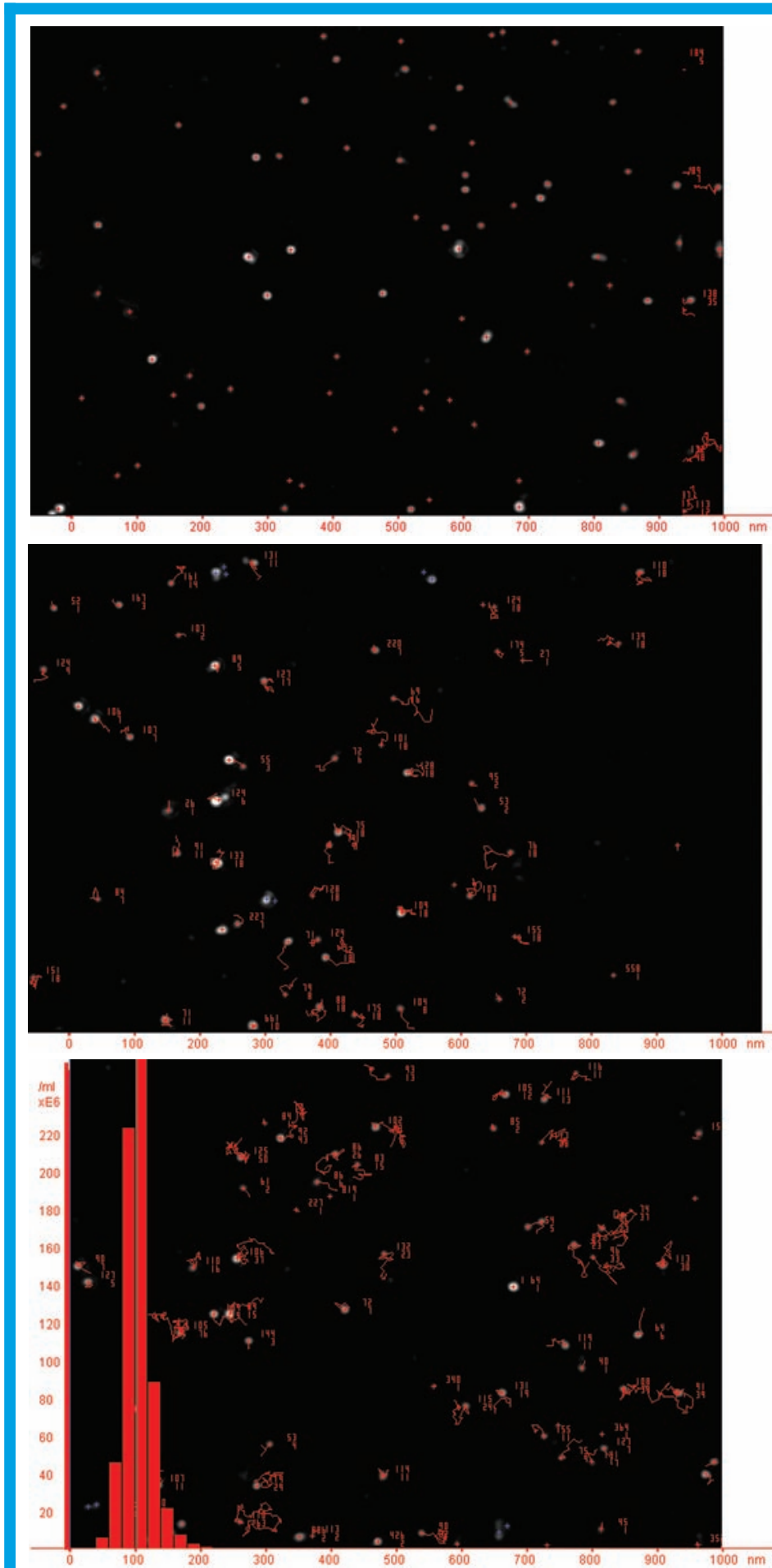

Figure 4, Video capture of particles.

Figure 5, Trajectory of individual particles.

Figure 6, Direct number count of particles.

The nanoparticles will move in the beam of the laser. They move randomly under solvent bombardment (Brownian motion) at a speed related to their size. The intensity of light scattered by a nanoparticle is related (through the power law) to its size. Small particles will move faster and further than large particles. Distance moved by each particle is measured and the average determined. The Particle Diffusion Coefficient is calculated. This relates to a sphere equivalent hydrodynamic diameter of the Stokes-Einstein equation.

Nanoparticles are too small to be imaged by the microscope. They are seen as point scatterers moving under Brownian motion.
Larger particles will scatter significantly more light than smaller particles while the speed of the particles varies strongly with particle size. The process of collection through to analysis is illustrated in these three images. figure 4 shows the video capture illustrating the Brownian motion. The second image, figure 5, shows the trajectory of each individual particle as derived form the series of CCD images captured over a few seconds. The third and final image, figure 6 , shows the final particle size distribution which is made up from all the separate measurements. The scales shown are linear. The horizontal scale runs from 1 to $1,000 \mathrm{~nm}$ while the vertical scale is a direct number count of particles, each one derived from an individual measurement.

\section{Comparison with PCS data}

When measuring a narrow range of particle sizes (e.g. a mono dispersion), PCS and NTA will deliver equivalent results. However, the strength of NTA is clearly observed when studying poly-dispersed samples where a range of sizes is present. If a sample containing two different sizes of particles is studied by PCS and by NTA, clearly different results are produced.

This is illustrated in a study of solution containing particles of $204 \mathrm{~nm}$ and $384 \mathrm{~nm}$ particles, figure $7 \mathrm{a}$ and $\mathrm{b}$. While the NTA data clearly shows the two sizes (a), the results from PCS only reports the larger size particle (b).

PCS cannot detect the smaller particles with the larger ones present because, as it measures light intensity, the results are dominated by the much greater scatter from the larger particles.
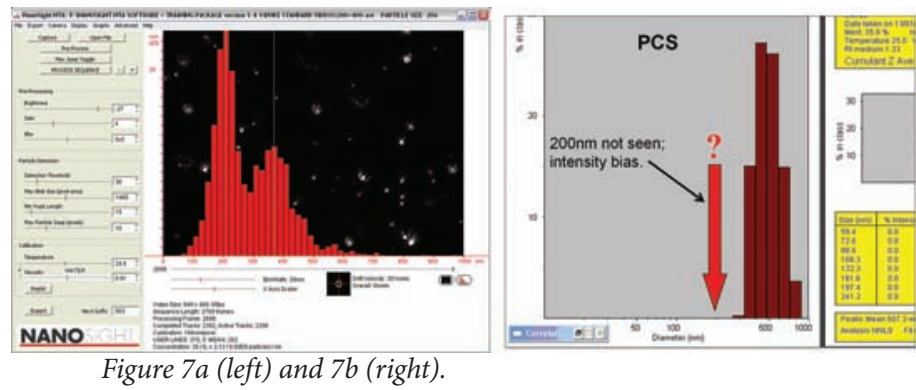

Applications for NTA

The breadth of samples that may be studied using NTA is extremely broad answering the particle sizing needs from fields as diverse as toxicology and materials science.

Nanoparticles are found in so many areas of research and industry today. For example, using liposomes as a drug deliverer allows potentially lower doses of drug to be used, reducing toxicity and side-effects. Furthermore, it is possible that gene therapy drugs may be delivered by liposomes. The size of the liposomes is increasingly being recognised as an important factor in treatment efficacy. The size of the liposome used in drug delivery may affect its circulation and residence time in the blood, the efficacy of the targeting, the rate of cell absorption (or endocytosis) and, ultimately, the successful release of its payload. Such size considerations are also hugely important to nanoscale polymer-encapsulated drug delivery systems. Accurate measurement of the particles being administered is therefore imperative. Figure 8.

In the oil and petroleum industry, the study of nanoparticulates may lead to increased knowledge of product efficiency. For example, lubricants as they age may suffer from the build-up of soot particles reducing the lubricant's effectiveness. NTA allows detection of soot particulates at a size well below that provided by optical microscopy, figure 9. Particles as small as $30 \mathrm{~nm}$ may be detected and the disper- 


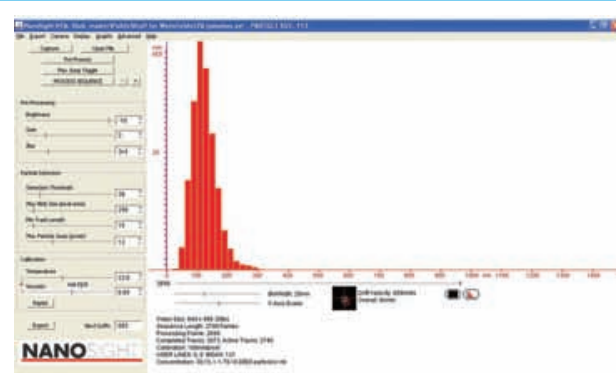

Figure 8, Sample of relatively mono-dispersed liposome with a modal size of $133 \mathrm{~nm}$.

sion assessed rapidly, in term of polydispersity, agglomeration and contaminant content.

Wear is critical in the development of artificial joints. Preclinical testing of orthopaedic implants have revealed critical information. The size of metallic, plastic or ceramic particles found in the synovial fluid can influence the immune system response to the particles and hence influence other factors such as remote particle deposition and third part particle wear. Figure 10.

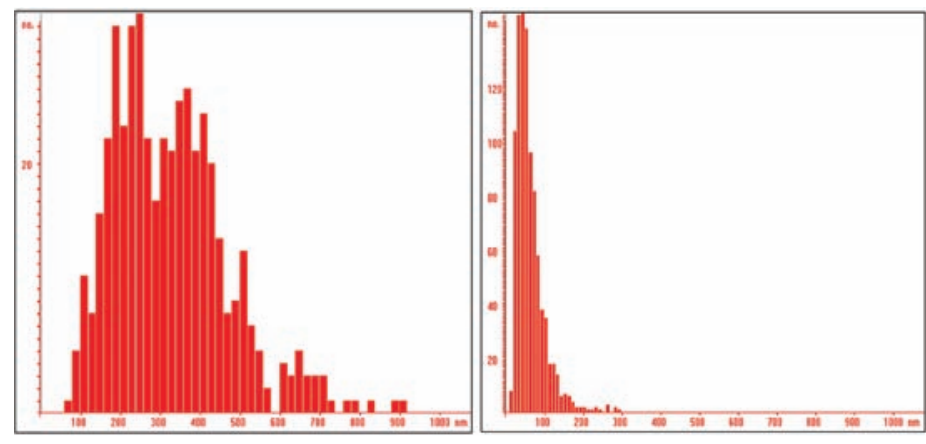

Figures $11 \mathrm{a}$ and $11 \mathrm{~b}$.

The study of inks and pigments may also be suited to NTA where the latest materials use much smaller particles than the original inkjet type of technology. Particle size is a core measurement in determining the functional properties of pigment-based inkjet inks. Colour density, opacity and viscosity depend directly upon particle size. Comparison of particle size distributions for two inks illustrates the ability to demonstrate polydispersity and the differences between them, figure $11 \mathrm{a}$ (left and fig $11 \mathrm{~b}$ (right). In both examples, the $\mathrm{x}$-axis represents particle diameterand the $y$-axes gives the number counts of particles at that size.

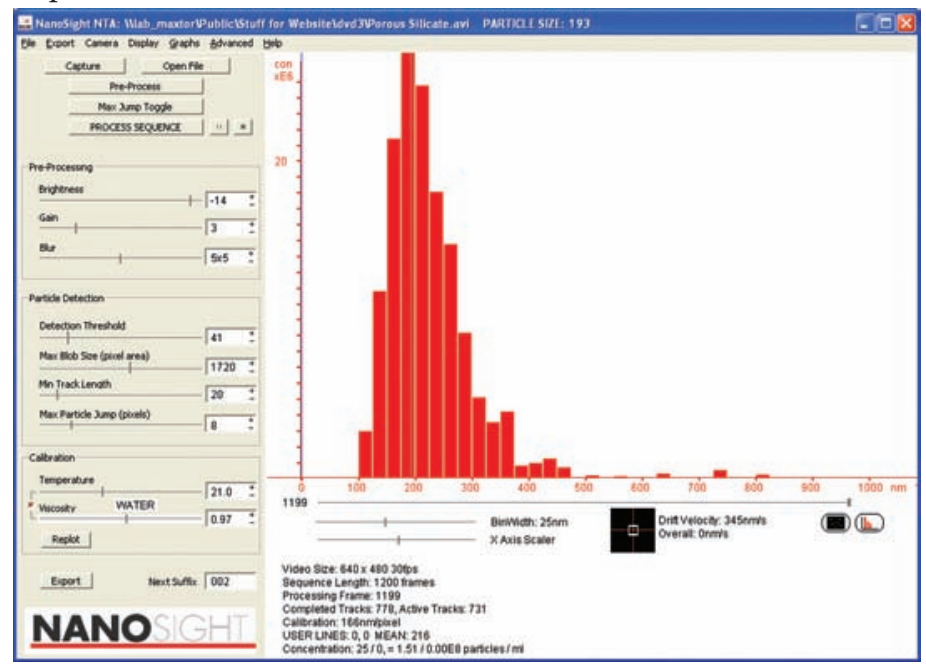

Figure 12, Results showing a generally mono-modal Dispersion of a porous silicate sample.

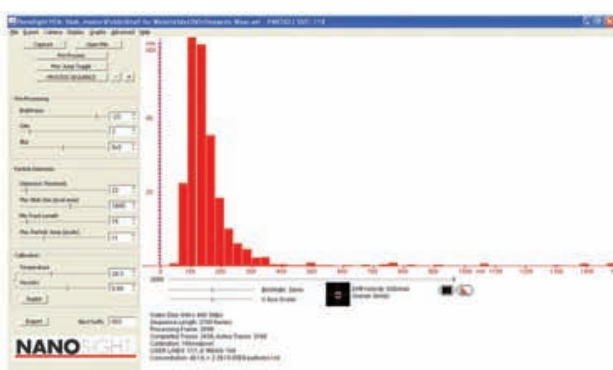

Figure 10, Distribution of particles found in synovial fluid surrounding an orthopaedic implant.

The final example described here looks at silicate nanoparticles used as a support for catalysts, figure 12. The size of the nanoparticles is critical as it determines the surface area of the catalyst. Furthermore, silicates may be functionalised with biological species, again an example where size is crucial to performance. With NTA, silicate particles may be characterised down to approximately $50 \mathrm{~nm}$ in size.

\section{Conclusions:}

The examples reported here have clearly shown the strength of the new technology of nanoparticle tracking analysis. Ideal for polydispersed nanoparticles in liquids, NTA offers significant benefits over the established techniques of PCS and electron microscopy. From the ease of sample preparation to the cost-of-ownership of the particle sizing system, NTA is rapidly gaining acceptance as a critical instrumentation technique for groups studying and applying nanoparticles in their work.

\section{PELCO ${ }^{\circledR}$ Silicon Nitride Membranes}

Next Generation $\mathrm{Si}_{3} \mathrm{~N}_{4}$ TEM Support Films with many advantages:

- Durable and chemically inert planar $50 \mathrm{~nm}$ substrate

- 3.0mm circular frame compatible with standard TEM holders

- EasyGrip ${ }^{\mathrm{TM}}$ micro rough edges for ease of handling

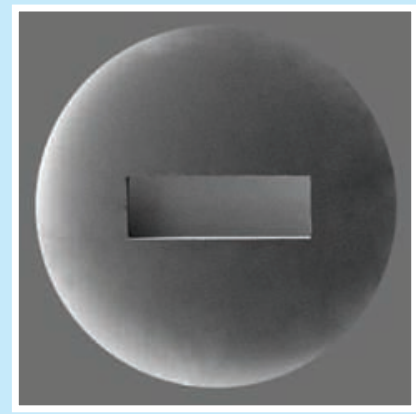

- Free from debris - no broken edges

- Large area support film: up to $0.5 \times 1.5 \mathrm{~mm}$

- Complimented with Aperture Frames and Blank Disks for nanotech experiments

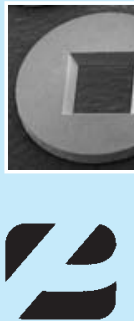

Aperture Frame

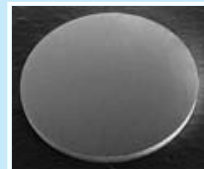

Blank Disks

\section{TED PELLA, INC.}

Microscopy Products for Science and Industry 